\title{
Acupuncture at Xinbaliao Point in Treating A Case of Urinary Retention after Rectal Cancer Surgery
}

\author{
Sa Zhang1, Gaiqin Yang2* \\ 1Shaanxi University of Traditional Chinese Medicine, Xianyang 712046, Shaanxi province, China \\ ${ }^{2}$ Shaanxi Provincial Hospital of Traditional Chinese Medicine, Xi'an 710003, Shaanxi Province, China \\ *Corresponding author: Gaiqin Yang, 564097200@qq.com
}

\begin{abstract}
Urinary retention after surgery is caused by inadvertent damage to the muscles and nerves around the bladder during the operation, which results in the suppression of the urination reflex, or the postoperative pain that causes sphincter spasm and dysuria. It is a common complication after anorectal disease, with an incidence rate of up to $52 \%$, which seriously affects the daily life of patients. It has been clinically discovered that acupuncture at Xinbaliao point can effectively improve urinary retention, relieve swelling in the lower abdomen, and improve the quality of life of patients. In this report, we present a case of postoperative urinary retention that had been treated with acupuncture at the Xinbaliao point.
\end{abstract}

Keywords: Acupuncture; Xinbaliao point; Urinary retention after surgery; Medical records

Publication date: July 2021; Online publication: July 30, 2021

A 68-year-old female patient was admitted to the hospital on February 16, 2021 due to postoperative urinary retention." Her chief complaint was difficulty urinating for 15 days after rectal cancer surgery. Based on her medical history, the patient had undergone "radical resection of rectal cancer" in Xijing Hospital 15 days ago.

After the surgery, the condition was stable and the residual urine volume was $200 \mathrm{~mL}$ under ultrasound examination, so indwelling catheterization was performed. The patient intended to seek acupuncture treatment in our department, and the outpatient is admitted to the hospital with the diagnosis of "urinary retention after surgery." The symptoms of admission include difficulty urinating, slight abdominal swelling, sore waist, fatigue, and sleepiness. Her tongue is light, the coating is thin and white, and the pulse is thin. Based on physical examination, her vital signs are stable. No abnormalities were observed in the heart and lungs. Four surgical scars about $2 \mathrm{~cm}$ in length were visible on the abdomen, the dressing was well wrapped, and the fistula bag was visible. There is no abnormality in the nervous system. In the auxiliary examination, the routine urine test suggests occult blood and high procalcitonin (PCT). Based on these findings, we consider urinary tract infection caused by indwelling catheterization. However, the patient had no symptoms such as fever, chills, urinary tract pain, etc., so no intervention was given, and she was advised to drink more water and put under close observation. The rest of the physical and chemical tests showed no obvious abnormalities. Western medicine diagnosis of this case is urine retention after surgery, whereas the Chinese medicine diagnosis is Fengshou ( $q i$ deficiency and blood stasis syndrome). Postoperative urinary retention is a common complication after anorectal disease, with an incidence rate of up to $52 \%{ }^{[1]}$.

Western medicine treatment is based on the principles of improving urinary motility, improving immunity, and preventing infection. Traditional Chinese medicine treatment is mainly based on acupuncture, replenishing $q i$ to remove blood stasis, promoting blood circulation and dredging collaterals. Acupoints such as Xinbaliao points (upper, second, middle, and lower), bilateral Shenshu, Qihaishu, 
Sanjiaoshu, Bladder Shu, Xuehai, Shuiquan, Zusanli, Sanyinjiao were selected. First of all, the patient took the prone position to expose the skin of the waist, buttocks and both lower limbs, and the acupoints were routinely disinfected. A $0.25 \mathrm{~mm} \times 75 \mathrm{~mm}$ filigree needle was used to puncture the Xinbaliao point, and the first needle (Zhongliao) was punctured at the side of the bend of the tailbone. The second stitch (lower lien) was punctured at 0.5 inch inward about one inch, and a diagonal line was drawn upwards at these two locations. The third stitch (secondary iliac) was punctured at 1 inch up from the middle liu, and go up 1 inch along the diagonal line of the third needle, and proceed to the fourth stitch (epi-liu). Four needles were placed on the left and right sides of the gluteal groove symmetrically, totaling 8 . The needle was in the shape of "inverted eight." The technique should be gentle, and applied with straight insertion. The needle should not be twisted, and the depth of the needle should be conducted to the lower abdomen due to the patient's conscious soreness or twitching. Then, several $0.25 \mathrm{~mm} \times 40 \mathrm{~mm}$ filigree needles were used to puncture the remaining acupoints, using routine methods of replenishing and reducing. The upper acupoints were kept for 30 minutes and treated once a day. Four groups of power distribution needles were used to dredge the meridians and collaterals, combined with waist infrared therapy to improve local blood circulation. After 1 week of treatment, the urinary tube was removed. The patient said she had increased desire to urinate, lower abdominal swelling disappeared, and waist soreness, drowsiness and fatigue were improved compared to the condition during admission. She also reported sleepiness, pale red tongue, thin white coating, and smooth pulse. The patient was instructed to hold the bladder properly and enhance the urination reflex after discharge from the hospital.

The main symptoms of this disease are urination, and lower abdomen swelling and pain. Therefore, it belongs to the category of "Long Shu" in Chinese medicine. The etiology and pathogenesis are mainly damp-heat betting, liver stagnation and $q i$ stagnation, deficiency of kidney $q i$ and urinary tract stasis, which lead to unfavorable triple energization, bladder opening and closing and loss of division. Urinary retention is a disease of bladder, which is closely related to the triple burner. In this case, the patient underwent surgical treatment for cancer, the internal organs were injured by a sharp blade, blood stasis was endogenous after the operation, the abdominal organs were damaged by the qi, the blood flow was weak, the new blood did not grow, the blood stasis did not go away, and the blood stasis blocked the bladder. The meridians, unfavorable $q i$ machine, loss of power to gasify the bladder, loss of power in opening and closing, and failure of water and wet transportation cause lower abdomen to swell. If a patient had difficulty in urination or defecation, he or she will become closed, and the obvious symptom is difficulty urinating. At this stage, for the treatment of this disease, Western medicines are mainly tamsulosin hydrochloride sustained-release capsules and neostigmine; traditional Chinese medicine mainly focuses on decoction, acupuncture, and acupoint injection ${ }^{[2]}$. Western medicine treatment needs to pay attention to its adverse reactions and contraindications, and acupuncture treatment is highly targeted, and it has no side effects.

The traditional Baliao points are located in the lumbosacral region, and are divided into upper, second, middle, and inferior points, which are opposite to the posterior foramen in turn. The acupuncture points named after "Liao" are generally some bone sutures or pores, and the pores are deep, which is difficult to accurately locate clinically. This is a difficult point in the treatment ${ }^{[3]}$. The new Baliao acupoint is an improved and innovative acupuncture point based on the traditional Baliao acupoint. Its position is lower than that of the traditional acupuncture point, and it is distributed along the tailbone in an "eight-character." From an anatomical point of view, Xinbaliao points can communicate internally and externally, regulate the nerve-humoral endocrine system, improve microcirculation, and regulate the tension of the pelvic floor and pelvic fascia, thereby regulating structure and function, and promoting muscle and nerve recovery. From the perspective of meridians, Xinbaliao point is located in the main path of the Zutaiyang bladder meridian. It can communicate with Chong and Ren, followed by the Governor Channel. Acupuncture can smoothen the bladder to promote the gasification function of the bladder and clear the waterway. From a 
holistic view of traditional Chinese medicine, acupuncture at Xinbaliao point can regulate yin and yang, mobilize the circulation of $q i$ and blood, make $q i$ and blood biochemically active, nourish damaged muscles and veins, and further promote the recovery of muscles and nerves around the bladder. At the same time, patients with hysterectomy are often accompanied by mental stress. The doctor should ease the patient's emotions and repeatedly do alternating exercises of abdominal muscle contraction and relaxation, which is conducive to regulating mind and promoting meridian-qi induction.

In summary, acupuncture at Xinbaliao acupoint has a significant effect on postoperative urinary retention. It is accurate in positioning and easy to operate, and should be promoted and used in clinical practice.

\section{Disclosure statement}

The authors declare that there is no conflict of interest.

\section{References}

[1] Yu Q, 2020, Analysis of the Causes of Urinary Retention and Preventive Measures after Anorectal Surgery. China Practical Medicine, 15(34): 162-164.

[2] Liu CC, Liu J, Yu H, et al., 2020, Research Progress in the Prevention and Treatment of Postoperative Urinary Retention in Anorectal Disease. Chinese Journal of Anorectal Disease, 40(12): 76-78.

[3] Wang YL, Wang LP, Shen JW, et al., 2020, Research Progress in Clinical Acupoint Selection and Operation of Baliao. Herald of Traditional Chinese Medicine, 26(14): 128-131, 137. 